\title{
Differentially Coherent Diversity Combining Techniques for DPSK Over Fast Rayleigh Fading Channels
}

\author{
Milica Stojanovic and Zoran Zvonar
}

\begin{abstract}
Performance of differential phase-shift keying (DPSK) is considered for fading channels with nonnegligible time-variation, as well as signal or noise correlation between diversity branches. A differentially coherent maximum-ratio combiner is derived, showing optimal weight dependence on the fading statistics. Bit error rate (BER) is evaluated analytically for Rayleigh fading, and comparison is made between the optimal and the equal-gain differentially coherent combiner. The optimal combiner provides improvement in all situations, and reduces the error probability floor. Performance improvement is higher for higher Doppler spreads, with the exact amount depending on diversity correlation. For low diversity correlation, equal-gain combining performance stays close to optimal.
\end{abstract}

Index Terms-Correlated diversity, differentially coherent detection, fast fading, maximum-ratio combining, mobile radio channel, Rayleigh fading, space-time processing.

\section{INTRODUCTION}

D IFFERENTIALLY coherent detection, together with diversity combining, offers a good tradeoff between receiver complexity and performance on Rayleigh fading channels [1]-[8]. The performance of differential phase-shift keying (DPSK) with diversity combining on Rayleigh fading channels is summarized in [1], where both equal-gain combining (EGC) and maximum-ratio combining (MRC) are addressed for a slowly fading channel. For rapidly fading channels, the impulse response of which cannot be assumed constant over the two consecutive symbol intervals needed for the detection of a DPSK symbol, receiver performance depends on the fading process temporal correlation. This dependence is analyzed in [2] for the case of equal-energy uncorrelated diversity. A related analysis for differentially encoded star-shaped QAM signals is given in [3].

In practice, a situation may arise in which there is correlation among diversity branches [1], resulting from insufficient channel spacing, be it frequency, time or space. In systems which exploit multipath diversity, receiver filtering may cause noise components to appear correlated at the input of the combiner. The same is true for the signal components, even if the physical channel exhibits uncorrelated scattering. Examples of correlated diversity include CDMA systems which exploit multipath diversity through rake filtering and

Manuscript received July 21, 1998; revised August 18, 1999.

M. Stojanovic is with the Department of Electrical and Computer Engineering, Northeastern University, Boston, MA 02115 USA.

Z. Zvonar is with Analog Devices, Wilmington, MA 01887 USA.

Publisher Item Identifier S 0018-9545(00)04833-7. multiuser detection [5], [6], as well as equalization problems for frequency-selective fading channels [7]. Ricean fading channels with trellis coded DPSK and block differential detection with correlated diversity is analyzed in [8]. Regardless of its origin, the correlation between diversity branches shall be termed "diversity correlation," which is to be distinguished from "temporal correlation" present within each of diversity branches due to the time-variation of fading processes.

Most commonly used diversity combining method for differentially coherent demodulation is EGC, in which the signals are added without weighting after differentially coherent demodulation. Such an approach offers simplicity of implementation, at the expense of a loss in performance caused by diversity correlation (due to receiver filtering, time-spreading, or co-channel interference) [4]. In a multipath diversity system [7], noise decorrelation was used to overcome the loss caused by receiver filtering, but the improvement was found to be subject to the Doppler spread of the channel.

The fact that DPSK diversity combining performance depends on both diversity correlation and temporal fading correlation motivates the design of a differentially coherent combiner which will use the knowledge of the fading channel statistics to provide performance improvement for all fading rates. Two questions immediately arise: How to design the combiner in an efficient manner, and what is the improvement available from this design, relative to the simple-to-implement EGC. The goal of the present analysis is to answer these questions.

In Section II, the optimal differentially coherent MRC for fast fading and correlated diversity is derived. The optimization criterion is maximization of the output signal-to-noise ration (SNR) when fading channel response can be estimated only implicitly, through differentially coherent detection. The probability of error for DPSK in terms of arbitrary combiner weights is obtained in Section III for the case of Rayleigh fading. Results are analyzed through numerical examples in Section IV, and conclusions are summarized in Section V.

\section{COMBINER OPTIMIZATION}

The signals observed at the input of diversity combiner after front-end receiver (matched) filtering at time $n T$, associated with the detection of the $n$th data symbol, are represented in complex baseband form as a column vector

$$
\mathbf{z}(n)=\mathbf{c}(n) d(n)+\xi(n)
$$




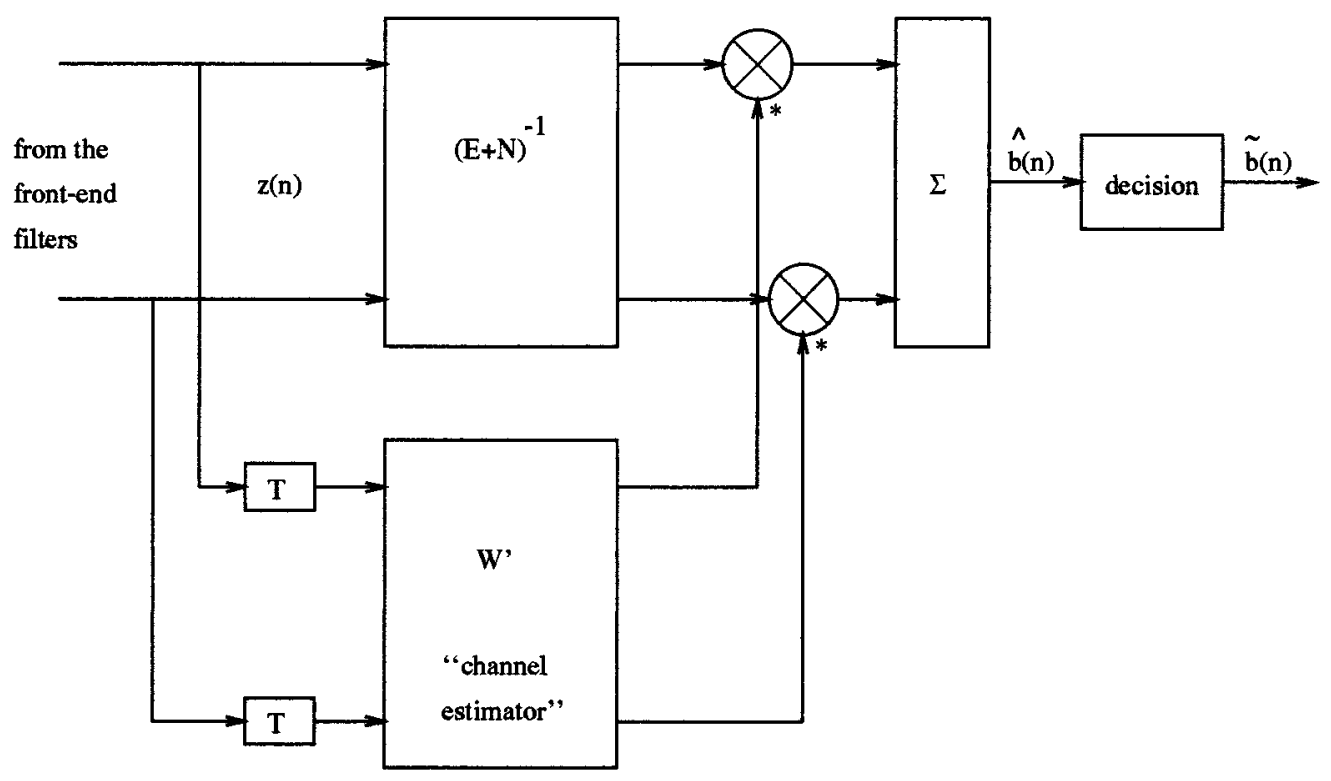

Fig. 1. Schematic representation of differentially coherent MRC.

where $\mathbf{c}(n)$ is the observed vector of fading channel gains, i.e., its discrete-time approximation, and $\xi(n)$ is the noise vector. The sequence of i.i.d. unit-variance data symbols $d(n)$ is obtained by differentially encoding the original sequence $b(n)$

$$
d(n)=b(n) d(n-1) .
$$

For M-DPSK, $b(n)=e^{j \Delta \phi(n)} ; \Delta \phi(n) \in\{0, \pm 2 \pi / M, \pm 2 \times$ $2 \pi / M, \ldots, \pi\}$.

The channel statistics are described by the correlation functions

$$
\mathbf{C}(m)=E\left\{\mathbf{c}(n+m) \mathbf{c}^{\prime}(n)\right\}, \quad m=0, \pm 1, \pm 2, \ldots
$$

where "prime" denotes conjugate transpose. In particular, the auto-correlation matrix $\mathbf{C}(0)$ describes signal correlation across diversity channels, while $\mathbf{C}( \pm 1)$ includes the effects of temporal correlation at the lag of one symbol interval. The noise vectors $\xi(n)$ are taken to be zero-mean, (temporally) white Gaussian, with covariance $\mathbf{N}$.

Differentially coherent combining of the signals $\mathbf{z}(n)$ can be represented in a general form

$$
\hat{b}(n)=\mathbf{z}^{\prime}(n-1) \mathbf{A} \mathbf{z}(n)
$$

where the matrix A contains the combiner weights. Several special cases can be distinguished. For equal-gain combining, $\mathbf{A}=\mathbf{I}$, i.e., no weighting is performed. ${ }^{1}$ When there is no correlation among diversities, in either the signal or the noise components, EGC is the optimal differentially coherent combining method. When the signal components are uncorrelated, but noise is correlated, the optimal MRC is given by $\mathbf{A}=\mathbf{N}^{-1}$ for channels with negligible time-variation. ${ }^{2}$

\footnotetext{
${ }^{1}$ The term EGC is not always consistently used. Some authors (e.g., [4]) refer to the above scheme as that of MRC, implying that weighting is implicitly performed by the delayed signals.

${ }^{2}$ This combining method can be interpreted as noise decorrelation followed by EGC (e.g., [7])
}

When the time-variation of the channels cannot be neglected, i.e., there is significant Doppler spreading, the MRC weights must depend on $\mathbf{C}(1)$ in addition to $\mathbf{C}(0)$ and $\mathbf{N}$. We refer to this combiner as the optimal MRC to signify the fact that maximization of combining ratio takes into account both the diversity correlation and the temporal correlation. To determine the optimal MRC combiner for this case, we view the process of differentially coherent combining as a special case of coherent combining in which the channel estimate is constrained to depend only on the delayed signal $\mathbf{z}(n-1)$ (and not on the previous values $\mathbf{z}(n-2)$, etc.). The receiver structure is shown in Fig. 1 . If $\mathbf{W}^{\prime}$ is the linear memoryless transformation used for channel estimation, the channel estimate $\hat{\mathbf{c}}(n)$ can be defined through

$$
\mathbf{W}^{\prime} \mathbf{z}(n-1)=\hat{\mathbf{c}}(n) d(n-1) .
$$

For a rapidly fading channel, the channel estimation error $\epsilon(n)=\mathbf{c}(n)-\hat{\mathbf{c}}(n)$ cannot be neglected in either differentially coherent or coherent combining [5]. It represents an additional noise term, which is assumed to be independent of the thermal noise (an assumption which is justified for all practical cases when the thermal noise is white and the channel is estimated from the previously observed signals). If the channel estimation error covariance is denoted by $\mathbf{E}$, the coherent MRC is given by

$$
\hat{d}(n)=\hat{\mathbf{c}}^{\prime}(n)[\mathbf{N}+\mathbf{E}]^{-1} \mathbf{z}(n) .
$$

Comparing (4) and (6), and using the implied relation

$$
\hat{d}(n)=\hat{b}(n) d(n-1)
$$

the differentially coherent MRC is interpreted as coherent combining in which the channel estimate satisfies (5), and the combiner matrix is

$$
\mathbf{A}=\mathbf{W}[\mathbf{E}+\mathbf{N}]^{-1} .
$$


Hence, a differentially coherent combiner can be designed in an efficient manner by choosing an appropriate channel estimator $\mathbf{W}$, and evaluating its error covariance ( $\mathbf{E}$ is a function of $\mathbf{W})$.

To specify the combiner, we focus on minimum mean squared error (MMSE) channel estimation. Unlike in the case of coherent MRC, the channel estimate is constrained by (5), or equivalently,

$$
\begin{aligned}
\hat{\mathbf{c}}(n) & =\mathbf{W}^{\prime} \mathbf{z}(n-1) d^{*}(n-1) \\
& =\mathbf{W}^{\prime}\left[\mathbf{c}(n-1)+d^{*}(n-1) \xi(n-1)\right] .
\end{aligned}
$$

The weights $\mathbf{W}$, which minimize the mean-squared error $E\left\{\epsilon^{\prime}(n) \epsilon(n)\right\}$, are given by

$$
\mathbf{W}=[\mathbf{C}(0)+\mathbf{N}]^{-1} \mathbf{C}^{\prime}(1) .
$$

The corresponding estimation error covariance is

$$
\mathbf{E}=\mathbf{C}(0)-\mathbf{C}(1)[\mathbf{C}(0)+\mathbf{N}]^{-1} \mathbf{C}^{\prime}(1) .
$$

On substituting the obtained values (10) and (11) into the expression (8), it is found that the optimal MRC/MMSE differentially coherent combiner is given by

$$
\begin{aligned}
\mathbf{A}= & {[\mathbf{C}(0)+\mathbf{N}]^{-1} \mathbf{C}^{\prime}(1)[\mathbf{C}(0)} \\
& \left.+\mathbf{N}-\mathbf{C}(1)[\mathbf{C}(0)+\mathbf{N}]^{-1} \mathbf{C}^{\prime}(1)\right]^{-1} .
\end{aligned}
$$

It may be pointed out that for a Rayleigh fading channel, in which $\mathbf{c}(n)$ is a complex, zero-mean Gaussian process, the same result can be obtained directly, using a different optimization criterion, namely maximization of the a posteriori probability density function of $\mathbf{z}(n)$ given $\mathbf{z}(n-1)$ and $b(n)$. Maximizing $p(\mathbf{z}(n) \mid \mathbf{z}(n-1), b(n))$ with respect to $b(n)=b$ is equivalent to maximizing its exponent factor

$$
\begin{aligned}
\max _{b}\left\{-\left[\mathbf{z}(n)-\mathbf{R}_{01} \mathbf{R}_{11}^{-1} \mathbf{z}(n-1)\right]^{\prime}\right. \\
\left.\quad\left[\mathbf{R}_{00}-\mathbf{R}_{01} \mathbf{R}_{11}^{-1} \mathbf{R}_{01}^{\prime}\right]^{-1}\left[\mathbf{z}(n)-\mathbf{R}_{01} \mathbf{R}_{11}^{-1} \mathbf{z}(n-1)\right]\right\}
\end{aligned}
$$

where $\mathbf{R}_{i, j}=E\left\{\mathbf{z}(n-i) \mathbf{z}^{\prime}(n-j) \mid b\right\}$ [9].

Using either criterion, the decision rule is given by

$$
\tilde{b}(n)=\arg \max _{b} \operatorname{Re}\left\{\hat{b}(n) b^{*}\right\}
$$

where $\hat{b}(n)$ is given by (4) and $b$ belongs to the set of transmitted $M$-ary DPSK symbols defined earlier.

\section{PRobability of ERRor ANALYSIS}

Bit error probability for binary DPSK is given by

$$
P_{e}=P\{\operatorname{Re}\{\hat{b}(n)\}<0 \mid d(n)=d(n-1)=+1\}
$$

which can be expressed in terms of a quadratic form as

$$
P_{e}=P\left\{\mathbf{g}^{\prime} \mathbf{J g}<0\right\}
$$

where

$$
\mathbf{g}=\left[\begin{array}{c}
\mathbf{A}^{\prime}[\mathbf{c}(n-1)+\xi(n-1)] \\
\mathbf{c}(n)+\xi(n)
\end{array}\right], \quad \mathbf{J}=\left[\begin{array}{ll}
\mathbf{0} & \mathbf{I} \\
\mathbf{I} & \mathbf{0}
\end{array}\right] .
$$

For a Rayleigh fading channel, $\mathrm{g}$ is a complex, zero-mean Gaussian vector, with covariance

$$
\mathbf{G}=\left[\begin{array}{cc}
\mathbf{A}^{\prime}[\mathbf{C}(0)+\mathbf{N}] \mathbf{A} & \mathbf{A}^{\prime} \mathbf{C}^{\prime}(1) \\
\mathbf{C}(1) \mathbf{A} & \mathbf{C}(0)+\mathbf{N}
\end{array}\right] .
$$

The probability (16) is then known to depend only on the eigenvalues of the matrix JG [1]. If the $I$ distinct eigenvalues of this matrix are denoted by $\left\{\lambda_{i}\right\}$, and each is of multiplicity $K_{i}$, the probability of error is given by

$$
P_{e}=\sum_{i: \lambda_{i}<0} \sum_{k=1}^{K_{i}} F_{i, k}
$$

where $\left\{F_{i, k}\right\}$ are the coefficients of partial fraction expansion of the characteristic function of the Gaussian quadratic form $G=\mathbf{g}^{\prime} \mathbf{J g}$

$$
C_{G}(j \omega)=\prod_{i=1}^{I} \frac{1}{\left(1-j \omega \lambda_{i}\right)^{K_{i}}}=\sum_{i=1}^{I} \sum_{k=1}^{K_{i}} \frac{F_{i, k}}{\left(1-j \omega \lambda_{i}\right)^{k}}
$$

Thus, the bit error probability is a function of combiner weights $\mathbf{A}$, as denoted by

$$
P_{e}=P(\mathbf{A}) .
$$

This result allows us to evaluate the performance of various combining methods for arbitrary diversity correlations and temporal correlations.

It may be interesting to note that when the optimal combiner (12) is used, the covariance $\mathbf{G}$ assumes a form identical to that found in coherent MRC with ideal MMSE channel estimation [5]. The difference, of course, is in the value of the error covariances $\mathbf{E}$ involved in differentially coherent and coherent combining. ${ }^{3}$

\section{EXAMPLES AND DISCUSSION}

To assess the combiner performance over a range of diversity correlations and for varying Doppler spreads, we concentrate on an example of binary DPSK with a second-order diversity and channel statistics that allow us to study the receiver performance as a function of two parameters only: one that describes temporal correlation, and one that describes energy distribution and correlations among diversity branches.

Assuming identical temporal correlation properties across diversity, we have that $\mathbf{C}(1)=\rho \mathbf{C}(0)$, where $\rho$ represents the one-step temporal correlation coefficient. It is related to the normalized Doppler spread $\omega_{d} T$ (normalized by the symbol rate $1 / T$ ), in a manner which depends on the shape of the Doppler spectrum. The fading process is often modeled as an auto-regressive Gauss-Markov process, or using the Jakes' model for land mobile radio channels. Coefficient $\rho$ as a function of $\omega_{d} T$ for several fading models can be found in [2].

${ }^{3}$ Ideal MMSE channel estimation for coherent combining is performed using all of the past values of the signal $\mathbf{z}(n)$. It can be accomplished by Kalman filtering if the fading processes obey an AR model whose parameters are known at the receiver. 

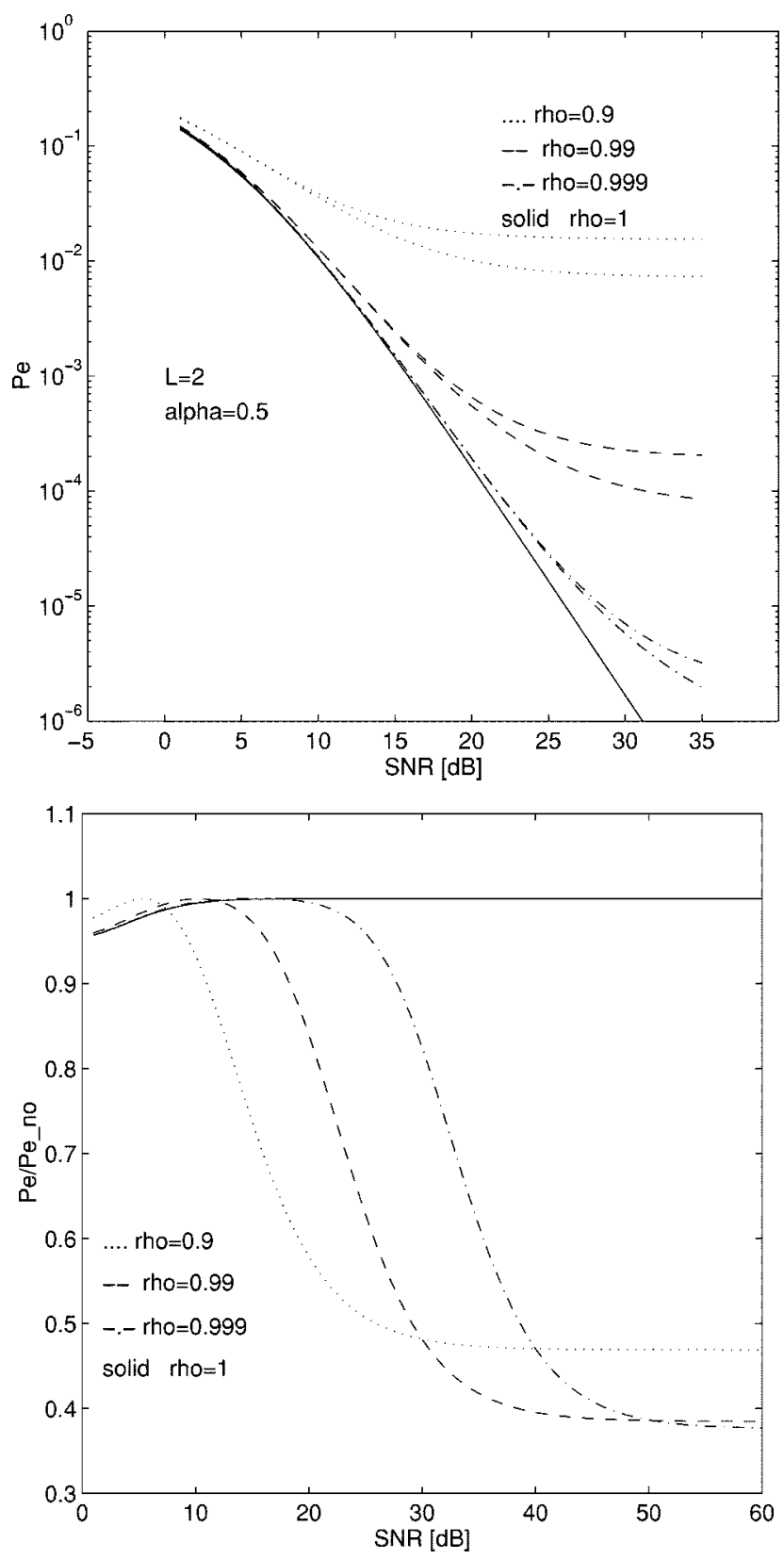

Fig. 2. Probabilities of error for combining with and without weighting, and their ratio as a function of $E_{b} / N_{0}$.

Correlation among diversity signals is described by

$$
\mathbf{C}(0)=\left[\begin{array}{cc}
1+(1-\alpha)^{2} & \alpha(1-\alpha) \\
\alpha(1-\alpha) & \alpha^{2}
\end{array}\right], \quad \alpha \in[0,1]
$$

This correlation matrix is constructed such that as the parameter $\alpha$ changes from 0 to 1 , the diversity changes from a single-order diversity for $\alpha=0$, through a range of second-order, correlated, unequal-energy diversities, to an uncorrelated, equal-energy diversity for $\alpha=1$. The noise is assumed to be uncorrelated, $\mathbf{N}=$ $N_{0} \mathbf{I}$. The SNR per bit is defined as SNR $=\operatorname{trace}[\mathbf{C}(0)] / N_{0}$.

Fig. 2 shows the bit error probabilities as functions of SNR with $\rho$ as a parameter, for correlated diversity described by $\alpha=$ 0.5 . For each value of $\rho$, two error probability curves are shown: the higher error rate corresponds to EGC $(\mathbf{A}=\mathbf{I})$ and the lower to the optimal MRC (12). The two curves apparently coincide only for $\rho=1$, i.e., for slow fading. The exact difference in performance depends on $\rho$, i.e., on the fading rate of the channel. While the difference is small at low SNR, with increasing SNR the loss of EGC rapidly increases, ultimately resulting in a higher error probability floor. The value $\rho=0.9$ is too low to be realistic for the majority of known applications; nevertheless it is included for the sake of completeness. On the other hand, using the Jakes' model, and assuming a vehicle speed of $80 \mathrm{mi} / \mathrm{h}$, a carrier frequency of $900 \mathrm{MHz}$, and a bit rate in the range $2400 \mathrm{~b} / \mathrm{s}-9600 \mathrm{~b} / \mathrm{s}, \rho$ is found to vary between 0.98 and 0.998 . For higher bit rates, such as $64 \mathrm{~kb} / \mathrm{s}, \rho$ stays close to 1 for a wide range of vehicle speeds $(\rho>0.9999$ for speeds below $150 \mathrm{mi} / \mathrm{h}$ ).

The second plot of Fig. 2 shows the ratio of the error probabilities obtained with and without combiner weighting, $P_{e} / P_{e, n_{o}}=P(\mathbf{A}) / P(\mathbf{I})$, with $\mathbf{A}$ chosen in the optimal manner, given by the expression (12). This ratio is clearly always less than one, demonstrating that the optimal MRC outperforms EGC uniformly, i.e., for all values of SNR. The difference in performance of the two combiners is caused both by diversity correlation and by fading dynamics. However, EGC loss caused by diversity correlation only (solid curves) is very small compared to that caused by the fading dynamics. In fact, it is practically nonexistent at higher SNR, and negligible at low SNR. As $\rho$ decreases, i.e., fading becomes faster, the gain obtained by matching the combiner parameters to the fading statistics starts to show at progressively lower SNR values. For example, with $\rho=0.99$, and SNR close to $30 \mathrm{~dB}$, the error probabilities differ approximately by a factor of two. The corresponding SNR loss is extremely high, as the error probability saturation region is almost reached for these parameters. At lower values of SNR, the loss of EGC is not severe at all, which justifies the use of this simple technique. (It should be noted, however, that additional performance degradation at finite SNR would result if there existed correlation between the noise components across diversity. Noise correlation, of course, has no effect on the error probability floor, which is caused by fading dynamics.)

Performance of the two combiners as a function of fading dynamics is summarized in Fig. 3. The parameter $\alpha$ is set to 0.3 , and the error probabilities of the two combiners are plotted versus $1-\rho$, which is proportional to the Doppler spread. The accompanying plot represents the ratio of the optimal MRC error probability to the error probability of EGC. Evidently, the optimal MRC outperforms the EGC uniformly for all values of $\rho$. Notably, performance improvement is present for practical values of $\rho$ in the range $0.999-0.9$. The error probability floors differ due to the signal correlations across diversity. (If instead of the signal, only the noise components were correlated, the two combiners would result in identical error floors.) At finite SNR and slow fading $(\rho \rightarrow 1)$, the performance of the two combiners is practically identical; however, at nonnegligible fading rates, the optimal combiner yields gain. The exact value of the gain depends on diversity correlations and the operating SNR.

The impact of diversity correlation is illustrated in Fig. 4 which shows the error probabilities, and their ratio, as a function of $\alpha$, at $\mathrm{SNR}=25 \mathrm{~dB}$. For slow fading, diversity correlation 

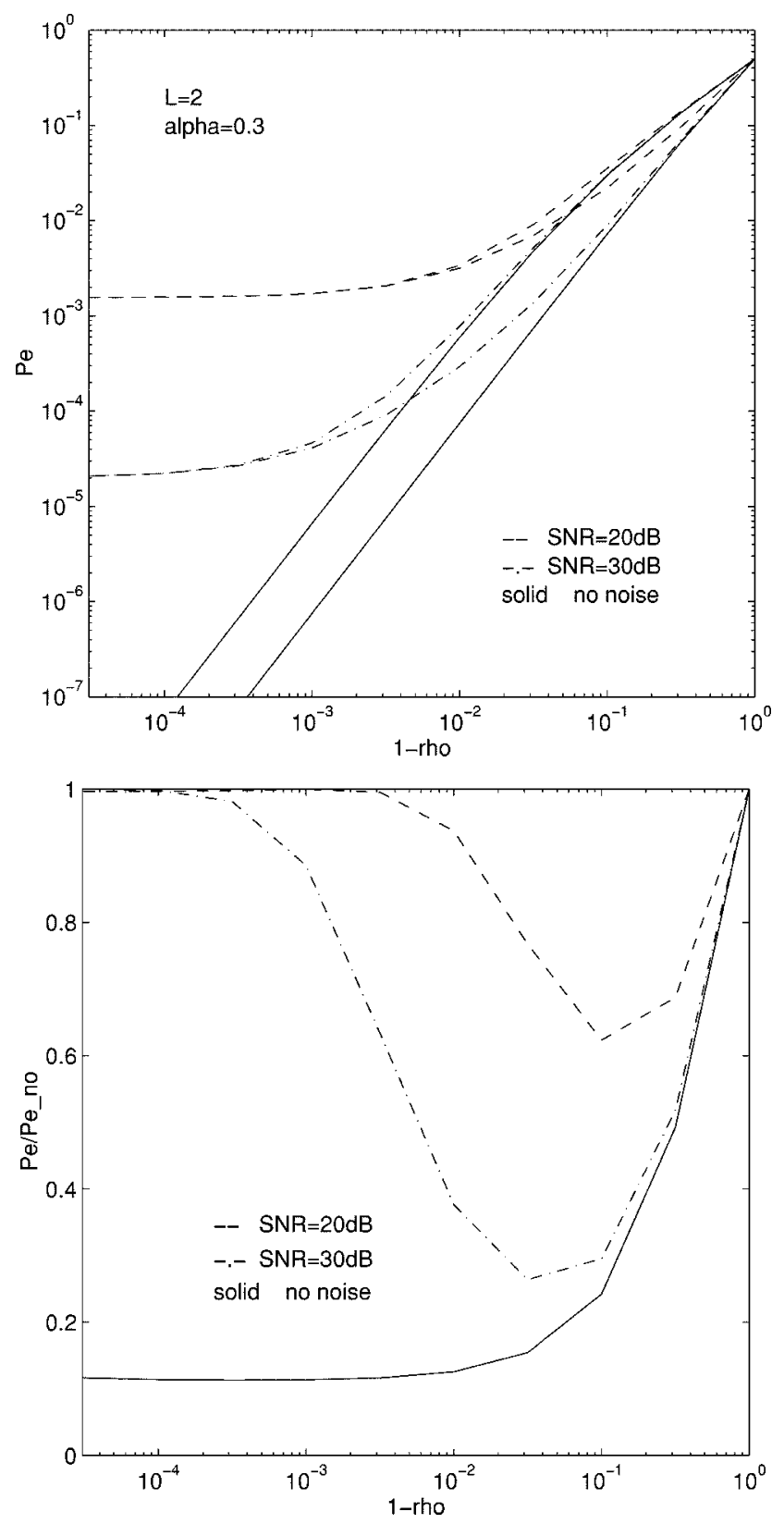

Fig. 3. Probabilities of error for combining with and without weighting, and their ratio as a function of $(1-\rho)$.

causes only a small difference in performance of the two combiners. However, as fading becomes faster, the optimal MRC results in a better performance for all but the limiting values of $\alpha=0$ and 1. (If in addition to the signal, the noise components were correlated too, the performance difference would exist as well at $\alpha=1$ ). Hence, the impact of frequency spreading, or fading dynamics, on the probability of error is emphasized by the presence of correlated diversity.

\section{CONCLUSION}

In summary, the optimal differentially coherent MRC provides an improvement over the differentially coherent EGC uniformly for all values of SNR, fading rates and varying degrees
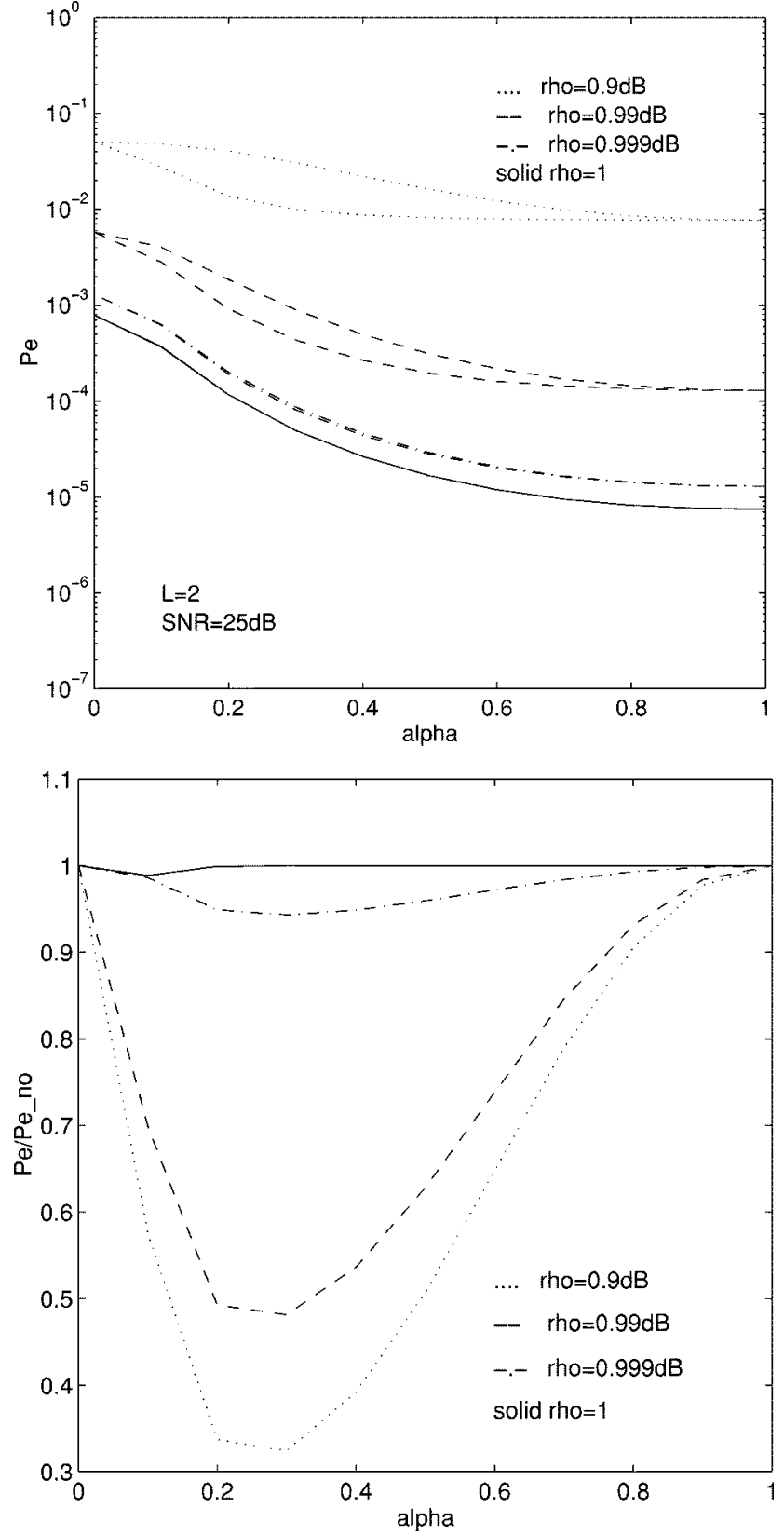

Fig. 4. Probabilities of error for combining with and without weighting, and their ratio as a function of diversity parameter $\alpha$.

of correlation among diversity branches with unequal energy distribution. The improvement is higher for higher fading rates, with the exact amount depending on the energy distribution and correlations across diversity branches. For practical values of SNR and realistic fading rates, the loss incurred by EGC can be kept small if diversity correlations are made relatively low.

Despite the fact that performance improvement is available from the knowledge of channel statistics, it is not likely that precise measurements of either the channel fading rates, or the signal and noise correlations will be available for the design of a practical MRC. The presented error probability analysis is not limited to any particular case, and it also provides a tool for evaluating the effects of parameter mismatch. 


\section{REFERENCES}

[1] M. Schwartz, W. R. Bennet, and S. Stein, Communication Systems and Techniques. New York: McGraw-Hill, 1966.

[2] P. Y. Kam, "Bit error probabilities of MDPSK over the nonselective Rayleigh fading channel with diversity reception," IEEE Trans. Commun., vol. 39, pp. 220-224, Feb. 1991.

[3] C. D. Chung, "Differentially amplitude and phase-encoded QAM for the correlated Rayleigh-fading channel with diversity reception," IEEE Trans. Commun., vol. 45, pp. 309-321, Mar. 1997.

[4] F. Adachi and K. Ohno, "BER performance of QDPSK with post-detection diversity reception in mobile radio channels," IEEE Trans. Veh. Technol., vol. 40, pp. 237-249, Feb. 1991.

[5] M. Stojanovic and Z. Zvonar, "Performance of linear multiuser detectors in time-varying multipath fading channels," in Proc. 5th Commun. Theory Mini-Conference (CTMC'96), London, U.K., Nov. 1996, pp. 163-167.

[6] M. Varanasi, "A systematic approach to noncoherent detection of DPSK modulation in single-user correlated diversity Rayleigh fading channels with application to post-combining decorrelative multiuser detection," presented at the Conf. Information Sciences and Systems, Baltimore, MD, Mar. 1997.

[7] M. W. Mydlow, S. Basavaraju, and A. Duel-Hallen, "Decorrelating detector with diversity combining for single user frequency-selective Rayleigh fading multipath channels," Int. J. Wireless Personal Commun., vol. 3, no. 1-2, pp. 175-193, 1996.

[8] J. Ventura-Traveset, G. Caire, E. Biglieri, and G. Taricco, "Impact of diversity reception on fading channels with coded modulation. Part II: Differential block detection," IEEE Trans. Commun., vol. 45, pp. 676-686, June 1997.

[9] A. D. Whalen, Detection on Signals in Noise. New York: Academic, 1971.

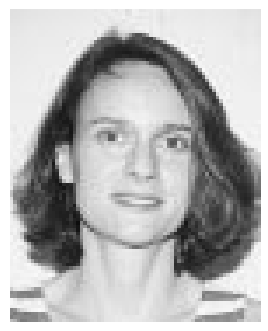

Milica Stojanovic received the Dipl.Ing. degree in electrical engineering from the University of Belgrade, Belgrade, Yugoslavia, in 1988, and the M.S. and Ph.D. degrees in electrical engineering from Northeastern University, Boston, MA, in 1991 and 1993, respectively.

She is a Visiting Professor at Northeastern University in Boston, MA, and a Guest Investigator at the Woods Hole Oceanographic Institution in Woods Hole, MA. Her research interests include digital communications theory and adaptive signal processing, with related problems in mobile radio and underwater acoustic communications.

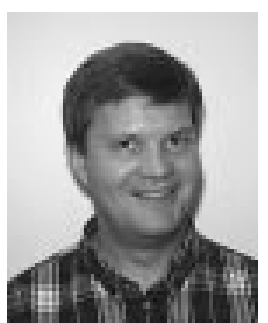

Zoran Zvonar received the Dipl.Ing. degree in 1986 and the M.S. degree in 1989, both from the Department of Electrical Engineering, University of Belgrade, Yugoslavia, and the Ph.D. degree in electrical engineering from the Northeastern University, Boston, in 1993.

From 1986 to 1989, he was with the Department of Electrical Engineering, University of Belgrade, where he conducted research in the area of telecommunications. From 1993 to 1994, he was a Postdoctoral investigator at the Woods Hole Oceanographic Institution, Woods Hole, MA, where he worked on multiple-access techniques for underwater acoustic communications. Since 1994, he has been with the Analog Device, Communications Division, Wilmington, MA, where he is the Manager of the Systems Development Group focusing on the design of wireless communications systems, with the emphasis on the integrated solutions and real-time software.

Dr. Zvonar was a Guest Editor of the IEEE TRANSACTIONS ON VEHICULAR TECHNOLOGY, the International Journal of Wireless Information Networks, and the ACM/Baltzer Wireless Networks, and a co-editor of the book GSM: Evolution Toward Third Generation Systems, (Norwell, MA: Kluwer, 1998). $\mathrm{He}$ is currently an Associate Editor of the IEEE COMMUNICATIONS LETTERS and a Feature Editor of the series on Software \& DSP in Radio in the IEEE COMMUNICATIONS MAGAZINE. 